\title{
A new energy consumption technique for mobile Ad-Hoc networks
}

\author{
Sameer Alani $^{1}$, Zahriladha Zakaria ${ }^{2}$, Herwansyah Lago ${ }^{3}$ \\ ${ }^{1,2}$ Faculty of electronic and computer engineering, Microwave Research Group (MRG), Centre for Telecommunication \\ Research and Innovation (CeTRI), Universiti Teknikal Malaysia Melaka (UTeM), Malaysia \\ ${ }^{3}$ Faculty of Engineering, University Malaysia Sabah, Malaysia
}

\begin{tabular}{l} 
Article Info \\
\hline Article history: \\
Received Aug 16, 2018 \\
Revised Apr 16, 2019 \\
Accepted Apr 27, 2019 \\
\hline Keywords: \\
Energy consumption \\
LOA \\
MANET \\
Quality of service \\
Routing protocol
\end{tabular}

Article Info

\begin{abstract}
A dynamic temporary network is created through wireless mobile nodes without the need for considerable infrastructure as well as a central manager. In a mobile ad hoc network, routing protocols allow a mobile for transmission and receiving packets. In the last decade, many variants have come up for the AODV. A minimum number of hop counts are chosen for enhancing routing protocols to include additional factors that can have an impact on path selections. As the distance between each node grows, the transmission power also rises accordingly. Hence, this impacts the network's entire performance and the most important feature is the quality of service. Most of the traditional routing protocols include energy consumption levels of the nodes and various parameters, like residual battery power, consumption of energy per packet and energy needed per transmission. A new technique is proposed in this paper to enhance the routing efficiency by making use of lion optimization algorithm after identifying all possible paths in the network. This technique not only enhances the energy efficiency of each node but also the performance metrics.
\end{abstract}

Copyright (c) 2019 Institute of Advanced Engineering and Science. All rights reserved.

Corresponding Author:

Sameer Alani,

Faculty of Electronic and Computer Engineering,

Universiti Teknikal Malaysia Melaka (UTeM),

Hang Tuah Jaya, 76100 Durian Tunggal, Melaka, Malaysia.

Email: itsamhus@gmail.com

\section{INTRODUCTION}

Currently, in the wireless communications field, wireless networks have become a key concern. These networks can be employed in different technology fields, like in industries, in the military as well as in personal area networks. Important attributes associated with wireless networks include cost-efficiency, reliability and easy installation, which are applicable to a broad range of applications [1]. Wireless networks like Wi-Fi networks, satellite communication systems and cellular phone networks do not require fixed infrastructure, which contrasts with wired networks.

Currently, mobile ad-hoc network (MANET) has gained wide popularity as a wireless ad hoc network [2]. It includes a set of mobile nodes that are independent of fixed infrastructure, like an access point or a base station [3]. A MANET is commonly employed for establishing communication in situations where common communication infrastructure is not available [4]. MANETs are employed in different applications, like in the military [5]. Since MANETs have a wide range of applications, these have become a hot spot in active research. Two functions are included in a network node: as a consumer and producer for data packet flow as well as a router for data packets made for other nodes [6].

However, two key challenges in MANET research are node mobility and limited battery life. MANET suffers from draining out energy and that could cause a crucial problem for the network [7]. 
When the node is out of energy, the routing is required to be restablished which also leads to consuming energy. Moreover, any collapse in the network affects the quality of service in the network in terms of throughput, packets loss and packets delivered. MANET's wireless topology can be altered rapidly and efficiently $[8,9]$. Moreover, the advantage of employing this kind of network is its capacity to function independently. The linking of a MANET can be done for the large-scale Internet. Since the 1990s, scholars have been using MANETs due to the growing popularity of Wi-Fi networks and laptop computers [9]. A MANET has two purposes in each node, in which each node can act as a source for sending information or turn out to be an intermediate node for other nodes for route information. In MANET, the nodes move freely and randomly, in which they can either join or leave the network at any point of time. The network's topology alters dynamically based on nodes' mobile nature. Thus, an appropriate routing protocol is needed to allow the network to adapt to the altering topology [10]. The issue that requires to be solved is how the ad hoc network routing changes to adapt with the generated complexity via subsequent movement for the network topology that keeps altering. Various routing protocols, together with dynamic source routing, ad-hoc on-demand distance vector routing (AODV) and destination sequenced distance vector routing, have been built [11].

There are three major types of routing protocols [12]: proactive, reactive and hybrid. For a network, the proactive routing protocols maintain an up-to-date topological map. For this mechanism, the routes are pre-identified and can be readily accessed when there is a need to send packets from the source to the destination. The availability of the route is intermittent in reactive routing protocols, which are also referred to as on-demand driven routing protocols. Therefore, query requests are sent to perform for a route discovery procedure, which flood the network when there is a need to send the data packet. The hybrid routing protocols integrate the advantages of both proactive and reactive routing protocols [13]. A proactive mechanism is employed by this type of routing when there is a node within the transmission range, which then switches to a reactive mechanism when the node is the present exterior of the transmission range. MANET routing can use numerous heuristic and artificial quality of service (QoS) routing algorithms. Routing can be segmented into various categories: source routing, single-and multi-path routing, which include the next step, i.e. centralized and distributed routing, hierarchical and flat routing, QoS-based and best-effort routing, queue-based routing, data- and address-centric routing, event-driven and energybased routing. This issue has been examined via numerous studies and has used employed methods, which are mostly artificial and heuristic intelligence tools.

\section{LITERATURE REVIEW}

MANET is a wireless network without a fixed infrastructure. Routing protocols link the nodes together to send and receive data from source to the destination. A regular power source is needed for each wireless node for node availability as well as effectiveness. To enhance the QoS, power efficiency and low MANET price need to be considered. To address these drawbacks in MANET, various methods were employed by researchers for modern wireless communication.

Hemalatha and Mahesh, n.d. put forward an enhanced algorithm method to determine the failure as well as Blac khole attacker nodes applicable for the network [14]. The algorithm employs directional antenna transmission for optimizing the consumed energy in MANET since the energy factor is a significant challenge. The simulation was done with the algorithm in NS2 and then compared with the protocol of the Ad-hoc On-Demand Distance Vector. The analysis result in which the proposed research algorithms were seen to perform almost $50 \%$ better when compared with the AODV protocol. Anand and Sasikala put forward another new method to minimize energy consumption in MANET. They employed the AODV protocol with enhanced routing strategy to give better quality during packet transmission [15]. This was achieved by NS2 simulation for their method, which demonstrated that their method gave better results than the currently employed power optimization strategy.

Deepa and Sutha [16] put forward a dynamic energy ad-hoc on-demand distance vector routing protocol (DE-AODV) for the packet delay in a bid to increase the network's lifetime and minimize the consumption of energy. The energy mechanisms are dependent on whether the mobile nodes are in the state of active, sleep or transmission. The DE-AODV protocol is employed for the determination of the nodes selection considered trustworthy and energy-efficient to choose the path route that is shortest from the source to destination. During packet transmission time, an external battery is employed for routing protocols should there be a chance of link failure. The proposed DE-AODV routing protocol gives better and effective output than with other currently employed techniques. Based on the simulation results, the proposed system gave better performance than when previous protocols were employed with regards to network stability rate, packet delivery ratio, end-to-end transmission, end-to-end delay of packets and throughput. 
These methods include Ant Colony Optimisation, Autonomous Localisation-based Eligible Energetic Path and Lion Optimisation Algorithm [11]. This method employed ALEEP scheme to measure the distance between the nodes, while ACO helps in setting the possible paths to initiate sending data from the source to the destination. The results demonstrated the effect of employing the ALLEP scheme; however, lack in maintenance phase continues to remain the issue as these nodes can be movable in the network.

Indexed and Vimala [17] put forward a novel bi-level optimization model to determine the optimal paths in mobile ad hoc networks to establish minimal energy consumption. A novel reliable bi-level optimization model employs a beakless path between nodes, while simultaneously considering the lowest energy consumption. This model uses all the information regarding the best fitness value for both the global best and local best. Finally, the determined results demonstrated the correctness of the proposed model. Indeed, a more stable route is achieved by selecting more energy conservative and shorter route but results in higher energy consumption.

\section{PROPOSED SOLUTION}

A lack of adequate energy to support mobile networking devices has become a major concern in MANETs. This shortage disrupts packet forwarding in MANETs. In MANET, energy consumption is considered a major issue and should be solved accordingly. Consequently, with a rise in the distance between nodes, the transmission power becomes greater correspondingly. When data are transmitted during the reception, a higher amount of energy is utilized.

Lion optimization algorithm (LOA) [18] is an optimization algorithm that has been introduced here. The simulation of the cooperative and solitary characteristics of lions is considered for building LOA, including mating, prey capturing, territorial marking, mutation, and defence. In this paper, the first technique known as LOA initialization is employed, which is an important process that not only safeguards the survival of lions but also allows randomly generating an initial solution. This strategy makes use of a route request for the initial path from the source to destination to get a fitness value as presented:

$$
\text { Lion }=\left[\mathrm{x}_{1}, \mathrm{x}_{2}, \mathrm{x}_{3}, \ldots \mathrm{x}_{\mathrm{n} \text { var }}\right]
$$

Cost (fitness value) of each lion is calculated by assessing the cost fitness as:

$$
\text { fitness value of lion }=\mathrm{f}(\text { lion })=\text { Lion }=\left[\mathrm{x}_{1}, \mathrm{x}_{2}, \mathrm{x}_{3}, \ldots \mathrm{x}_{\mathrm{n} \text { var }}\right]
$$

The proposed technique employs a new routing technique that considers the lion optimization algorithm (LOA) for identifying the best path selection between the destination and the actual node source. This is a different strategy in the LOA, which is heading towards a safe place signified as mobility for the network nodes. For each lion, the best path is selected as the best solution, which is considered a significant path from the source to destination to initiate sending data along with mobility in the network and then adjust transmission power accordingly for every node. The LOA starts identifying when adjustment of the transmission power for all nodes is completed, as presented in Figure 1.

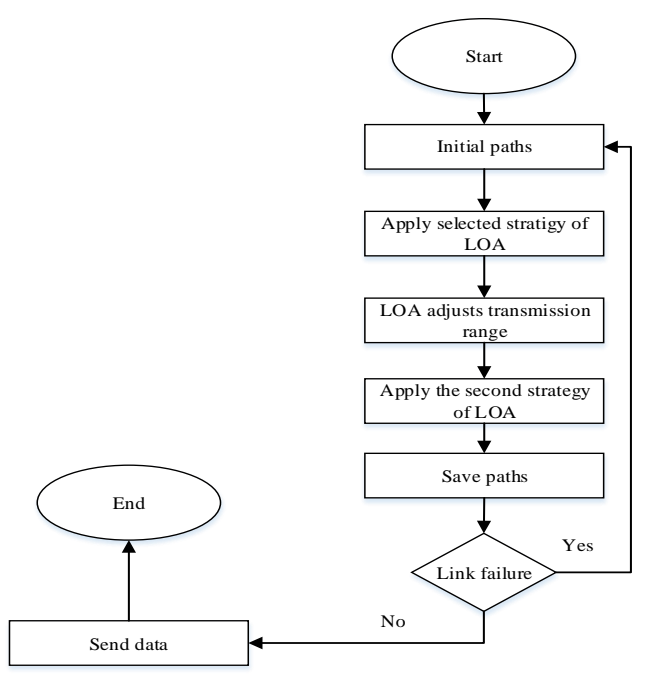

Figure 1. The proposed solution 
Initially, a route request (RREQ) packet is broadcasted by the source node $S$ to its 1-hop neighbor nodes by considering the $\mathrm{K}$ values list. A list of 1-hop neighbor $\mathrm{K}$ values is present for the routing table (RT) entry for each node. With the help of this RREQ packet, destination D is explored by each node and the search continues for entries of D in its RT until identification of all possible paths. The source node stores the best path in its memory. The newly generated path is now the current best path, and transmission of data initiates simultaneously with it. Figure 1 illustrates the overall method.

\section{SIMULATION PARAMETERS}

The simulation has done by MATLAB. Different parameters used in the simulation with one scenario which is the node velocity. Table 1 demonstrates the different parameters.

Table 1. The parameters of the simulation

\begin{tabular}{cc}
\hline Parameter & Value \\
\hline Node velocity & $1,2,3,4,5$ \\
Packet size & 64 bytes \\
Simulation time & $100 \mathrm{Ms}$ \\
Traffic type & CBR \\
\hline
\end{tabular}

\section{PERFORMANCE METRICS}

The different metrics utilized to assess the performance of the recommended method are:

\subsection{Packet Delivery Ratio}

Packet delivery ratio (PDR) is the data packets ratio delivered to the destination node for the data packets generated by the source [19]. This metric signifies the quality of the routing protocol when data packets are delivered from the source to destination. The packet delivery ratio is calculated through the equation:

$$
\text { Packet delivery ratio }=\frac{\text { Lpackets received by destination }}{\sum \text { packets sent by sources }} *
$$

\subsection{Remaining Energy}

The residual energy for each node is the remaining energy [20]. All the residual energy of each node is summed up to calculate the total remaining energy.

$$
E_{\text {tot }}=\sum_{i=1}^{n} E_{i}
$$

where $\mathrm{Et}_{\mathrm{ot}}$ is the total remaining energy of the network, whereas $\mathrm{E}_{\mathrm{i}}$ is the remaining energy of $\mathrm{i}^{\text {th }}$ node

\subsection{Packets Dropped}

This can be defined as the difference in ratio between the number of data packets received and the number of data packets sent when a packet is received by a router during processing of another packet. Storing of the received packet should be done in the input buffer that is waiting its turn. The input buffer of the router has a limited size [21]. A time would come when the capacity of the buffer is full, and then the next packet is forced to be dropped. In the network, the impact of packet loss is the need for the packet to be resent, which could result in overflowing and lead to more packet loss. A good metric would be to observe paths followed with the help of our algorithm. This metric has demonstrated us the path reliability selected by our algorithm. Packet drop can be determined as follows.

$$
P D(\%)=\frac{\text { No of packets sent }- \text { Noof packet received }}{\text { No of packet sent }} \times 100
$$

\section{RESULTS AND ANALYSIS}

\subsection{Remaining energy}

The changed in remaining energy occurs due to increased node speed. On increasing the node speed to $1,2,3,4$ and $5 \mathrm{~m} / \mathrm{s}$, a decrease is seen in the remaining energy. When the rise in nodes' speed causes an increase in the probability for the link to fail, the protocol will need to initiate a new path from the source to the destination node. This process uses a substantial amount of energy, even though the proposed approach 
gives higher performance when compared with other approaches. This is because the proposed approach uses a stable path that has the highest energy level. Figure 2 shows the results of the new method and AODV.

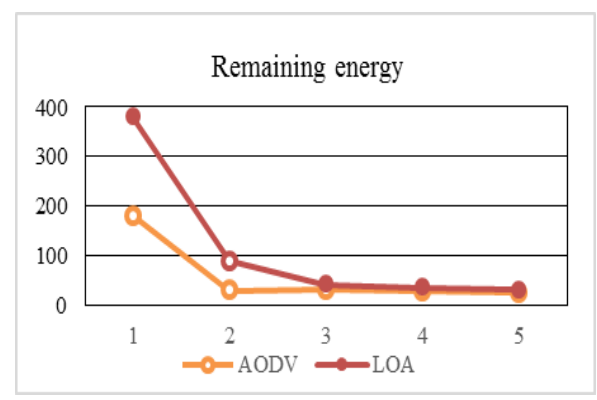

Figure 2. Remaining energy

\subsection{Packet delivery ratio}

The change in PDR when raising the nodes' speed is shown in Figure 3. While there is an increase in node speed to $1,2,3,4,5 \mathrm{~m} / \mathrm{s}$, a decrease in PDR is observed. Also, the LOA approach is reduced and the AODV energy is dropped simultaneously. The results demonstrate that a negative impact is cast by the node speed on the PDR for all protocol types. However, when compared with the AODV protocol, the proposed technique gave better PDR due to the generation of the best path between the source and destination nodes. With this technique, the packet delivered to the destination is increased.

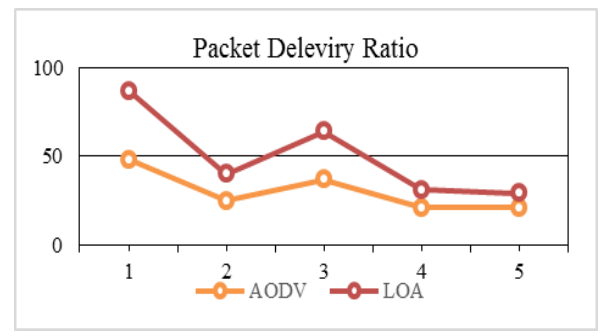

Figure 3. Packet delivery ratio

\subsection{Packet drop ratio}

Figure 4 demonstrates the change in packets dropped with an increase in the nodes' speed. While there is an increase in node speed to $1,2,3,4,5 \mathrm{~m} / \mathrm{s}$, there is a rise in packets drop. When the rise in the node velocity, the results of AODV are dropped. LOA is impacted, and more packets are dropped because of the high velocity. However, the results demonstrate that the proposed technique provides better performance with regards to packet drop when compared with both AODV. This is because the proposed technique chooses the shortest and highest energy path from the source to destination nodes. This results in a stable path, which in turn decreases the packets drop.

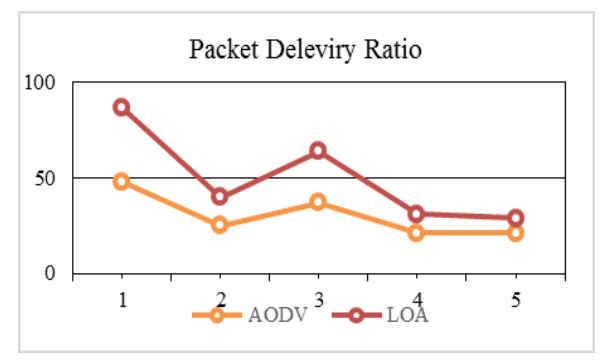

Figure 4. Packet drop ratio 


\section{CONCLUSION}

A network developed by nodes and which functions in an environment a fixed infrastructure is known as an ad-hoc network. In this case, a routing protocol examination is vital for message transmission routing. Routing protocols are classified as on-demand, table-driven, or an amalgam of table-driven routing protocols. Messages can be transmitted by routing instantly as and when communication is required through regular communication. LOA has been selected and deployed because of its alluring strategies. The outcomes indicated the prowess of the recommended network. Going forward, research studies can emphasize on the security concerns in MANET.

\section{ACKNOWLEDGEMENTS}

The authors would also like to thank Center for Research and Innovation Management (CRIM), UTeM's research grant JURNAL/2018/FKEKK/Q00001 and Universiti Teknikal Malaysia Melaka (UTeM) for their encouragement and help in supporting this work

\section{REFERENCES}

[1] M. N. Abdulleh, et al., "Comparative Study of Proactive, Reactive and Geographical MANET Routing Protocols," Commun. Netw., vol/issue: 07(02), pp. 125-137, 2015.

[2] P. Singh and H. Dhawan, "Node mobility based route selection in AODV for use in MANETs," Proc. - 1st Int. Conf. Comput. Commun. Control Autom. ICCUBEA 2015, pp. 83-87, 2015.

[3] M. Aamir and M. A. Zaidi, "A buffer management scheme for packet queues in MANET," Tsinghua Sci. Technol., vol/issue: 18(6), pp. 543-553, 2013.

[4] S. Giordano, "Mobile Ad-Hoc Networks," 2000.

[5] A. Fongen, et al., "A Military Mobility Model for MANET Research," IASTED Int. Conf. Parallel Distrib. Comput. Networks, 2009.

[6] P. Goyal, et al., "MANET: Vulnerabilities, Challenges, Attacks, Application," IJCEM Int. J. Comput. Eng. Manag. ISSN, vol. 11, pp. 2230-7893, Jan 2011.

[7] A. Taj, et al., "Optimized Model for Energy Aware Location Aided Routing Protocol in MANET," vol/issue: 12(14), pp. 4631-4637, 2017.

[8] H. S. H. Jassim, et al., "Scenario based performance analysis of reliant ad hoc on-demand distance vector routing (R-AODV) for mobile ad hoc network," vol. 2, pp. 78-89, May 2011.

[9] J. Godwin and R. Srinivasan, "A Survey on MANET Security Challenges,, Attacks and its Countermeasures," Int. J. Emerg. Trends Technol. Comput. Sci., vol/issue: 3(1), pp. 274-279, 2014.

[10] S. A. K. A. Omari and P. Sumari, "An Overview of Mobile Ad Hoc Networks for the Existing Protocols and Applications," Int. J. Appl. Graph Theory Wirel. Ad Hoc Networks Sens. Networks, vol. 2, pp. 87-110, 2010.

[11] S. A. Hussein and D. P. Dahnil, "A New Hybrid Technique to Improve the Path Selection in Reducing Energy Consumption in Mobile AD-HOC Networks," vol/issue: 12(3), pp. 277-282, 2017.

[12] M. K. Nazir, et al., "A Novel Review on Security and Routing Protocols in MANET," Commun. Netw., vol/issue: 08(04), pp. 205-218, 2016.

[13] M. H. A. Wahab, et al., "A Technical Review of MANET Testbed Using Mobile Robot Technology," J. Phys. Conf. Ser., vol. 1049, pp. 012001, 2018.

[14] S. Hemalatha and P. C. S. Mahesh, "Energy Optimization in Directional Advanced Intruder Handling AODV Protocol in MANET," vol. 14, pp. 935-960, 2004.

[15] M. Anand and T. Sasikala, "Efficient energy optimization in mobile ad hoc network (MANET) using better-quality AODV protocol," Cluster Comput., pp. 1-7, 2018.

[16] J. Deepa and J. Sutha, "A new energy based power aware routing method for MANETs," Cluster Comput., pp. 1-8, Jan 2018.

[17] S. Indexed and S. Vimala, "Multicast Optimal Energy Aware Routing Protocol for Manet Based on Swarm Intelligent Techniques," vol/issue: 8(1), pp. 967-975, 2017.

[18] M. Yazdani and F. Jolai, "Lion Optimization Algorithm (LOA): A nature-inspired metaheuristic algorithm," J. Comput. Des. Eng., vol/issue: 3(1), pp. 24-36, 2016.

[19] A. Tsirigos and Z. J. Haas, "Analysis of Multipath Routing - Part I: The Effect on the Packet Delivery Ratio," IEEE Trans. Wirel. Commun., vol/issue: 3(1), pp. 138-146, 2004.

[20] L. M. Feeney, "An energy consumption model for performance analysis of routing protocols for mobile ad hoc networks," Mob. Networks Appl., vol/issue: 6(3), pp. 239-249, 2001.

[21] W. Wang and M. Linderman, "Defending against Collaborative Packet Drop Attacks on MANETs," 2nd Int. Work. Dependable Netw. Comput. Mob. Syst. (DNCMS 2009), pp. 1-6, 2009. 


\section{BIOGRAPHIES OF AUTHORS}

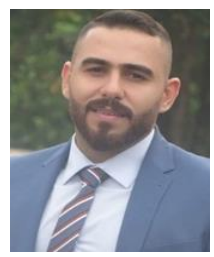

Sameer Alani was born in Iraq, in 1989. He received the B.S. degree in computer engineering and the M.Sc. degree in wireless communication and Computer networking technology from The National University of Malaysia (UKM), in 2017. He is currently pursuing the Ph.D. degree in wireless communication and networking. His research interests include antenna applications, wireless communication and networking Technology.

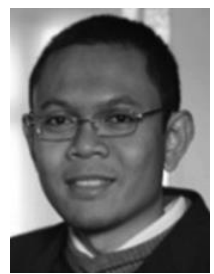

Zahriladha Zakaria was born in Malaysia in 1975. He received the B.Eng. and M.Eng. degrees in electrical and electronic engineering from Universiti Teknikal Malaysia Melaka (UTeM), in 1998 and 2004, respectively, and the Ph.D. degree in microwave engineering from the University of Leeds in 2010. From 1998 to 2002, he was with STMicroelectronics, Malaysia, where he was a Product Engineer. He is currently a Professor and a Coordinator with the Centre for Telecommunication Research and Innovation, Faculty of Electronic and Computer Engineering, (UTeM), where he teaches electronic system, communication principles, microwave engineering, wireless communications, and signal processing. His research interests include variety of microwave devices development, such as planar and non-planar microwave filters, resonators, amplifiers, and antennas. He also investigates data communication and radio wave propagation in wireless communication systems. He has published more than 100 scientific papers in journals, proceedings, and book-chapters. He holds eight intellectual property rights and he has received several awards, including Gold Medal during several research and innovation exhibitions at the national and international level, such as UTeMEX 2012, 2013 and 2015, Malaysia Technology Expo (MTE 2012-2014 and 2016), ITEX 2016, the International Trade Fair Ideas Inventions New Products (iENA 2012), Nuremberg, Germany, and the Seoul International Invention Fair (SiiF 2013), Seoul, South Korea.

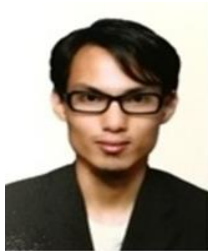

Herwansyah Lago was born in Sabah, Malaysia, on 26 August 1990. He received the B. Eng and PhD degrees in Communication Engineering from Universiti Malaysia Perlis (UniMAP), Perlis, Malaysia, in 2013 and 2017, respectively. In 2017, he was appointed as a Post-Doctoral Research Fellow in Universiti Teknikal Malaysia Melaka (UTeM), Melaka, Malaysia. He is currently working as a lecture in Universiti Malaysia Sabah (UMS). His research interest focusses on electronic telecommunication especially in antenna structure, metamaterials, dielectric materials, RF and microwave design. 